\title{
Overriding of the preseptal orbicularis oculi muscle in Caucasian cadavers
}

\author{
Hirohiko Kakizaki ${ }^{1,2}$ \\ Weng Onn Chan ${ }^{2}$ \\ Yasuhiro Takahashi ${ }^{3}$ \\ Dinesh Selva ${ }^{2}$
}

'Department of Ophthalmology, Aichi Medical University, Nagakute, Aichi, Japan; ${ }^{2}$ South Australian Institute of Ophthalmology and Discipline of Ophthalmology and Visual Sciences, University of Adelaide, Australia; ${ }^{3}$ Department of Ophthalmology and Visual Sciences, Osaka City University Graduate School of Medicine, Osaka, Japan
Correspondence: Hirohiko Kakizaki Department of Ophthalmology, Aichi Medical University, Nagakute, Aichi 480-I 195, Japan

Tel +8I $56 \mid 6233$ II (Ext. 218I)

Fax+8I 56I 637255

Email cosme@dI.dion.ne.jp

\begin{abstract}
We aimed to microscopically examine whether Caucasian eyelids demonstrate overriding of preseptal orbicularis oculi muscle (OOM) over the pretarsal OOM in both lower and upper eyelids. Full thickness sections of 13 lower eyelids and 11 upper eyelids from seven Caucasian cadavers were examined. In the lower eyelids, all 13 specimens demonstrated clear overriding of preseptal OOM over the pretarsal OOM. The overriding part extended almost to the level of lower eyelid margin. However, in the upper eyelids, only one of the 11 eyelids demonstrated overriding, and the overriding part only extended to the level of mid-tarsal plate. Our result strongly supports the hypothesis of overriding of the preseptal OOM over the pretarsal $\mathrm{OOM}$ as an etiology of involutional lower eyelid entropion. The relatively low frequency of upper eyelid overriding preseptal OOM in our study reflects and may explain the rare occurrence of involutional upper eyelid entropion.
\end{abstract}

Keywords: Caucasian, overriding, orbicularis oculi muscle, preseptal, pretarsal

The etiology of involutional lower eyelid entropion has been hypothesized to be a combination of horizontal and longitudinal lower eyelid laxity, ${ }^{1-4}$ and overriding of the preseptal orbicularis oculi muscle (OOM) over the pretarsal part of the OOM. ${ }^{5}$ Examination of lower eyelid laxity is relatively easy and can be achieved by several known methods, ${ }^{6,7}$ in contrast, examination of overriding preseptal OOM is difficult. ${ }^{8}$ Entropion surgery such as Wheeler's procedure, ${ }^{9}$ where horizontal tightening of the OOM results in resolution of entropion provides indirect evidence to the hypothesis that overriding of OOM as an etiology of involutional entropion. However evidence in support of this hypothesis remains scarce and therefore the role of overriding OOM in the etiology of involutional lower eyelid entropion is still controversial. ${ }^{10-12}$

Overriding of preseptal OOM over the pretarsal OOM has been shown in microscopic cadaveric study of Asian lower eyelids to be a typical feature in Asian eyelids. ${ }^{13}$ This feature is thought to influence the fuller appearance of Asian lower eyelid, and possibly a causative factor of involutional lower eyelid entropion. ${ }^{13}$ None of the eyelids in this study demonstrated entropion even though cadaveric eyelid tends to loosen horizontally secondary to loss of basal muscular tone. ${ }^{14}$ For Caucasian lower eyelids, there has been no microscopic cadaveric studies, ${ }^{15}$ magnetic resonance imaging (MRI), or any other imaging studies ${ }^{16,17}$ done for overriding of preseptal OOM. In addition, previous imaging or microscopic cadaveric studies in the upper eyelid were not focused on the overriding of the preseptal OOM on the pretarsal part, and overriding has not been identified as a causative factor of involutional upper eyelid entropion. ${ }^{18}$

The purpose of this study was to microscopically examine Caucasian cadavers to determine whether both lower and upper Caucasian eyelids demonstrate any overriding of preseptal OOM over the pretarsal OOM. 


\section{Materials and methods}

The process of procuring the eyelid samples has been previously described. ${ }^{13,19}$ During dissection, none of the cadavers demonstrated entropion in both the lower and upper eyelids. Full thickness sections of 13 lower eyelids (7 right and 6 left) and 11 upper eyelids ( 7 right and 4 left) from seven Caucasian cadavers (age range: $78-101$ years old at death; age average: 87.7 years old) were fixed in $10 \%$ buffered formalin. Sagittaly sliced sections of the central part were stained with Masson's Trichrome and examined under the microscope. Micrographs were taken with a digital camera system attached to the microscope.

All the cadavers were registered with the University of Adelaide, and proper consents and approvals were obtained prior to use. Methods for securing human tissues were humane and complied with the tenets of the Declaration of Helsinki.

\section{Results}

In the lower eyelids, all 13 specimens demonstrated clear overriding of the preseptal OOM over the pretarsal OOM (Figure 1). The overriding part extended almost to the level of lower eyelid margin. However, in the upper eyelids, 10 eyelids did not show any overriding (Figure 2), and only one of the 11 eyelids demonstrated overriding (Figure 3 ) in which the overriding part only extended to the level of mid-tarsal plate.

\section{Discussions}

Overriding of the preseptal OOM over the pretarsal OOM in the lower eyelid was present in all the Caucasian cadavers, but all but one did not show any overriding in the upper eyelid.

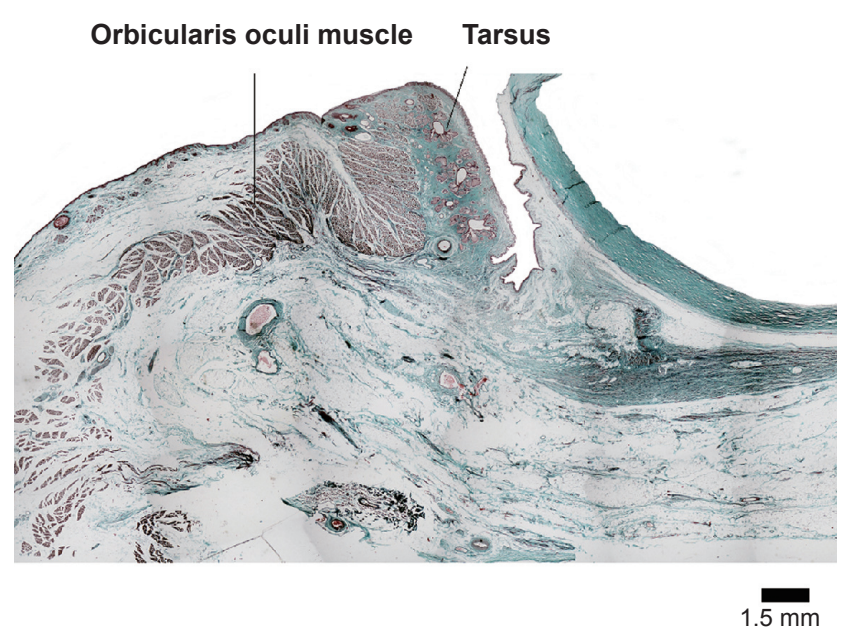

Figure I The preseptal orbicularis oculi muscle of the lower eyelid overrides over the pretarsal orbicularis oculi muscle. Bar $=1.5 \mathrm{~mm}$.
The finding in the lower eyelids is similar to that from previous studies on Asian lower eyelids. ${ }^{13}$ We believe this overriding to be a common feature between races in cadaveric lower eyelids.

As cadavers are usually kept without head support and take up a chin-up position, lower eyelid preseptal OOM may be easily moved toward the eyelid margin and override the pretarsal OOM due to effects of gravity. On the contrary, gravity pushes the pretarsal OOM of the upper eyelid cranially, and may explain why all upper eyelids but one did not show the overriding of the preseptal OOM to the pretarsal part of the OOM.

The condition of the lower eyelid in the dead is similar to that of involutional lower eyelid entropion. In both states, the lower eyelids are loosened horizontally. The lower eyelid in the dead allows for the movement of OOM towards the eyelid margin. As vertical laxity did not seem to present in the cadavers and the movement of OOM is thought to have occurred slowly and less dynamically, they may have prevented the manifestation of lower eyelid entropion. Despite this, the presence of overriding preseptal OOM over the pretarsal OOM was clearly elucidated. Thus, this finding strongly supports the hypothesis that overriding of the preseptal OOM could be an etiology of involutional lower eyelid entropion. In addition, as all but one of the upper eyelids did not show any overriding, this finding reflects and may explain the rare occurrence of involutional upper eyelid entropion. ${ }^{18}$

The overriding part of the lower eyelid extended almost to the level of lower eyelid margin, but the overriding part in the upper eyelid extended to the level of mid-tarsal plate. Considering the effects of gravity, the lower eyelid is pushed anteriorly by the orbit, but the upper eyelid is

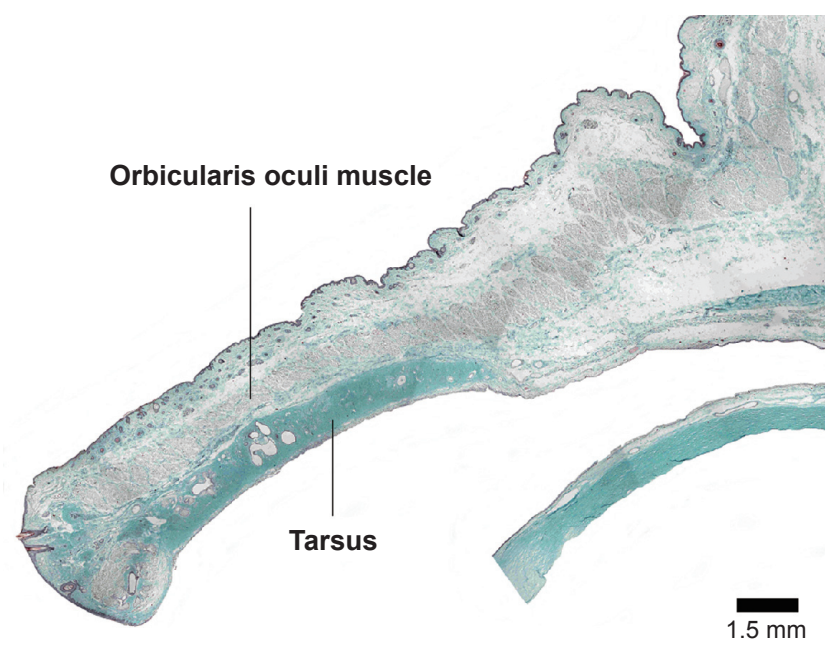

Figure 2 The preseptal orbicularis oculi muscle of the upper eyelid continues straightly to the pretarsal orbicularis oculi muscle. Bar $=1.5 \mathrm{~mm}$. 


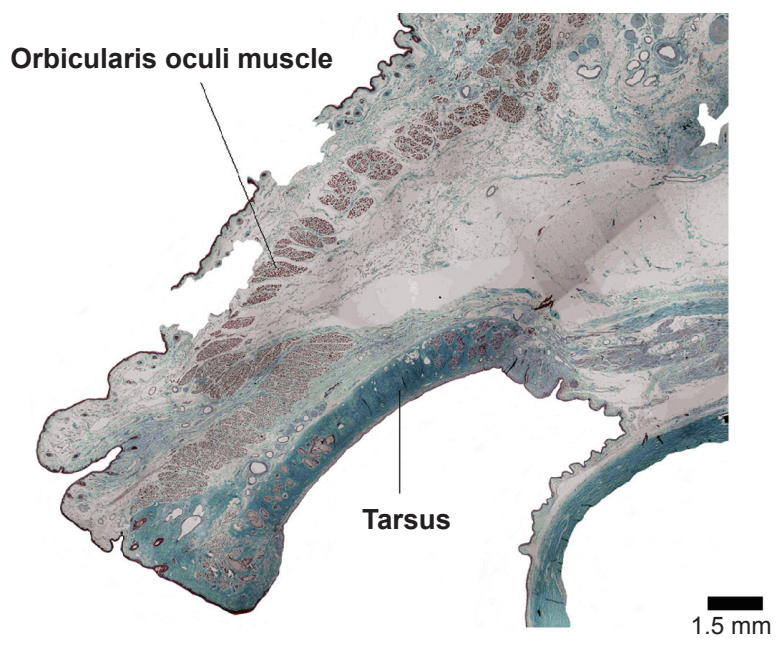

Figure 3 The preseptal orbicularis oculi muscle of the upper eyelid overrides over the pretarsal orbicularis oculi muscle. Bar $=1.5 \mathrm{~mm}$.

pushed posteroinferiorly. This concept originates from understanding the pathophysiology of "post-enucleation socket syndrome". ${ }^{20}$ In this situation, the lower edge of the lower tarsal plate is pushed anterosuperiorly, but the upper edge of the upper tarsal plate is pushed posteroinferiorly. Therefore, in the lower eyelid, overriding of the preseptal part over the pretarsal part can effectively invert the lower eyelid margin, but in the upper eyelid, as the overriding part and the rotation axis is far from the upper eyelid margin, involutional upper eyelid entropion seldom occurs. ${ }^{18}$

Overriding of the preseptal over the pretarsal OOM has not been the focus in previous MRI studies of the eyelid. ${ }^{16,17}$ Therefore even though some MRI scans of Asian eyelids in the literature ${ }^{16}$ showed overriding preseptal OOM, the finding was not reported. However, even in the presence of involutional lower eyelid entropion, preseptal overriding is difficult to observe in vivo. We believe this is because the force needed to produce overriding preseptal OOM may have be used instead to invert the lower eyelid margin and thus it manifests as entropion instead of observable overriding preseptal OOM.

Although overriding preseptal OOM is demonstrated in both Caucasians and Asians, ${ }^{13}$ there remain several differences in lower eyelid anatomy between both groups. One major difference is the orbital fat in Asians extends anterosuperiorly to the inferior border of tarsal plate, ${ }^{16}$ but in Caucasians the orbital fat extends superiorly only to the point of confluence of the capsuloplapebral fascia with the orbital septum, ${ }^{21}$ approximately $5 \mathrm{~mm}$ below the inferior tarsal border. ${ }^{18}$ This difference may be why Caucasians suffer from much less involutional lower eyelid entropion than Asians. ${ }^{21}$
The shape of OOM is a little different between generations. ${ }^{22}$ In the young specimen of the lower eyelid, the muscle bundles of the pretarsal OOM are located in a line along the tarsus and made a U-turn toward the skin, giving the muscle an $\mathrm{S}$ shape. The muscular shape of the pretarsal OOM, resembling a chrysanthemum petal, is compact to provide movement to the tarsus. The bundle shape of the pretarsal OOM is quite different compared with that of the preseptal OOM. In the aged specimen of the lower eyelid, the pretarsal muscles are joined together and S shape was less clear. In addition, the preseptal OOM is pressed forward and its thickness is reduced by herniation of the orbital fat. The involutional lower eyelid entropion may be caused by these involutional changes.

The drawbacks of this study are only sampling the old-age cadavers and in vivo samples could not be used. However, it was very difficult to take young cadavers, and we were obliged to take cadaveric study as the current findings were difficult to observe in vivo.

In conclusion, all 13 lower eyelids specimens and only one of the 11 upper eyelids demonstrated clear overriding of preseptal over the pretarsal OOM. The overriding part extended almost to the level of lower eyelid margin but only to the level of mid-tarsal plate in the upper eyelid. These results strongly support the hypothesis that overriding of the preseptal over the pretarsal OOM is one of the etiologies of involutional lower eyelid entropion. This may explain the rare occurrence of the involutional upper eyelid entropion.

\section{Disclosure}

The authors report no financial support and no financial interest related to this manuscript.

\section{References}

1. Jones LT, Reeh MJ, Tsujimura JK. Senile entropion. Am J Ophthalmol. 1963;55:463-469

2. Collin JRO, Rathbun JE. Involutional entropion. A review with evaluation of procedure. Arch Ophthalmol. 1978;96:1058-1064.

3. Benger RS, Musch DC. A comparative study of eyelid parameters in involutional entropion. Ophthal Plast Reconstr Surg. 1989;5:281-287.

4. Haefliger IO, Piffaretti JM. Lid retractors desinsertion in acquired ptosis and lower lid entropion. Klin Monatsbl Augenheilkd. 2001;218: 309-312.

5. Danks JJ, Rose GE. Involutional lower lid entropion. To shorten or not to shorten? Ophthalmology. 1998;105:2065-2067.

6. Fante RG, Elner VM. Transcaruncular approach to medial canthal tendon plication for lower eyelid laxity. Ophthal Plast Reconstr Surg. 2001;17:16-27.

7. Olver JM, Sathia PJ, Wright M. Lower eyelid medial canthal tendon laxity grading: an interobserver study of normal subjects Ophthalmology. 2001;108:2321-2325.

8. Kakizaki H, Zako M, Mito H, et al. Magnetic resonance imaging of pre- and postoperative lower eyelid states in involutional entropion. Jpn J Ophthalmol. 2004;48:364-367. 
9. Wheeler JM. Spastic entropion correction by orbicularis transplantation. Trans Am Ophthalmol Soc. 1938;36:157-162.

10. Jones LT. The anatomy of the lower eyelid and its relation to the cause and cure of entropion. Am J Ophthalmol. 1960;49:29-36.

11. Hurwitz JJ. Senile entropion: the importance of eyelid laxity. Can $J$ Ophthalmol. 1983;18:235-237.

12. Jackson ST. Surgery for involutional entropion. Ophthalmic Surg. 1983;14:322-326.

13. Kakizaki H, Jinsong Z, Zako M, et al. Microscopic anatomy of Asian lower eyelids. Ophthal Plast Reconstr Surg. 2006;22:430-433.

14. Henssge C, Lunkenheimer PP, Salomon O, et al. Supravital electrical excitability of muscles. Z Rechtsmed. 1984;93:165-174.

15. Hawes MJ, Dortzbach RK. The microscopic anatomy of the lower eyelid retractors. Arch Ophthalmol. 1982;100:1313-1318.

16. Carter SR, Seiff SR, Grant PE, et al. The Asian lower eyelid: a comparative anatomic study using high-resolution magnetic resonance imaging. Ophthal Plast Reconstr Surg. 1998;14:227-234.
17. Galatoire O, Touitou V, Heran F, et al. High-resolution magnetic resonance imaging of the upper eyelid: correlation with the position of the skin crease in the upper eyelid. Orbit. 2007;26:165-171.

18. Miller DG, Hesse RJ. Involutional entropion of the upper lid. Ophthal Plast Reconstr Surg. 1990;6:16-20.

19. Kakizaki H, Zako M, Nakano T, et al. The levator aponeurosis consists of two layers that include smooth muscle. Ophthal Plast Reconstr Surg. 2005;21:379-382.

20. Smit TJ, Koornneef L, Zonneveld FW, et al. Computed tomography in the assessment of the postenucleation socket syndrome. Ophthalmology. 1990;97:1347-1351.

21. Carter SR, Chang J, Aguilar GL, et al. Involutional entropion and ectropion of the Asian lower eyelid. Ophthal Plast Reconstr Surg. 2000;16:45-49.

22. Iwanami M, Tsurukiri K. Histological comparison between young and aged specimens of the oriental lower eyelid using sagittal serial sections. Plast Reconstr Surg. 2007;119:2061-2071. 